\title{
The Diagnostic Dilemma of Ruptured Liver Metastasis in a Patient with Lung Cancer A case report
}

K.S. Jayanth, ${ }^{1}$ Shivakumar Madan, ${ }^{1}$ Balakrishnan Gurushankari, ${ }^{1}$ Sathasivam Sureshkumar, ${ }^{1}$ Amaranathan Anandhi, ${ }^{1}$ Rajesh N.G., ${ }^{2}$ "Vikram Kate ${ }^{1}$

\begin{abstract}
Spontaneous rupture of a metastatic liver tumour is rarely documented in the literature when compared to hepatocellular carcinoma and other liver lesions, especially from a lung primary. We report a case of ruptured liver metastasis from an adenocarcinoma of the lung mimicking ruptured liver abscess, challenging the clinical diagnosis. A 42-year-female patient presented to a tertiary care institute in 2020 with complaints of abdominal pain, breathlessness and fever. On examination, the patient was tachypnoeic with a right hypochondriac mass. A contrast-enhanced computed tomography of abdomen and thorax revealed an ill-defined heterogeneously enhancing lesion in the liver with a communicating subcapsular collection and hypo-enhancing lesions in the left lobe and heterogeneously enhancing lesion in the left lung. Adenocarcinoma of the lung with hepatic metastasis was confirmed with a core needle biopsy. The patient was managed conservatively with intravenous antibiotics, intercostal drainage tube and gefitinib. However, despite best efforts, the patient succumbed to the disease.
\end{abstract}

Keywords: Metastasis; Spontaneous Rupture; Hepatocellular Carcinoma; Thyroid Transcription Factor; Liver Abscess; Case Report; India.

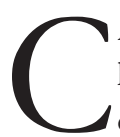

ARCINOMA OF THE LUNG IS ONE OF THE lethal cancers with the commonest sites of dissemination being brain, liver, contralateral lung, adrenals and bone. ${ }^{1,2}$ Although rupture of liver lesions such as liver abscess, hepatic adenomas, haemangiomas and focal nodular hyperplasia have been reported in the literature, rupture of malignant tumours is relatively uncommon. ${ }^{3}$ Spontaneous rupture of hepatocellular carcinoma (HCC) is reported in approximately $10 \%$ of cases due to its hypervascularity and decreased coagulation factors as a result of underlying liver cirrhosis. ${ }^{4,5}$

However, spontaneous rupture of a metastatic liver tumour is rarely documented in the literature when compared to $\mathrm{HCC}$ and other liver lesions., ${ }^{4,5}$ Rupture of liver metastasis in a patient with lung cancer is even more rare with isolated cases mentioned in the literature. We report a case of ruptured liver metastasis from an adenocarcinoma of the lung presenting with subcapsular collection and massive pleural effusion mimicking a ruptured liver abscess, associated with portal and hepatic venous thrombosis, challenging the clinical diagnosis. To the best of the authors' knowledge, no such case has been reported.

\section{Case Report}

A 42-year female patient, a homemaker from a village of South India, was brought by ambulance to the Emergency Department of a tertiary care hospital in 2020 with complaints of dull-aching right upper abdominal pain, breathlessness (modified Medical Research Council grade 3) and intermittent high-grade fever for 15 days. ${ }^{6}$ The patient had a history of loss of appetite and significant weight loss over two months. The patient did not have any history of vomiting, jaundice, prior hospitalisation or surgeries. She had no known medical comorbidities. She gave a history of usage of firewood as fuel for cooking purposes in an ill-ventilated house for the past 25 years. She had no history of tobacco or alcohol consumption.

On examination, the patient was afebrile, anaemic, anicteric and breathless with a respiratory rate of $26 / \mathrm{min}$. The patient's blood pressure was 110/70 $\mathrm{mmHg}$ and her pulse rate was $110 / \mathrm{min}$. Abdominal examination revealed an $8 \times 6 \mathrm{~cm}$ soft, ill-defined, tender mass in the right hypochondrium. On digital rectal examination, no growth or deposits were palpable. On auscultation of bilateral lung fields, reduced breath sounds were heard in both infra-mammary, infra-axillary and infrascapular areas, with no added respiratory sounds, possibly suggesting pleural effusion. Preliminary blood investigations showed haemoglobin of $9.1 \mathrm{~g} /$ $\mathrm{dL}$ and leukocytosis of 15,510 cells $/ \mathrm{mm}^{3}$ with normal renal parameters and electrolytes. Liver function tests showed normal bilirubin and transaminase levels with only a mild elevation of alkaline phosphatase (228 $\mathrm{IU} / \mathrm{L})$.

Chest radiograph, ultrasound (USG) abdomen and contrast-enhances computed tomography (CECT) of abdomen and thorax was carried out [Table 1]. The CECT of her abdomen and thorax 


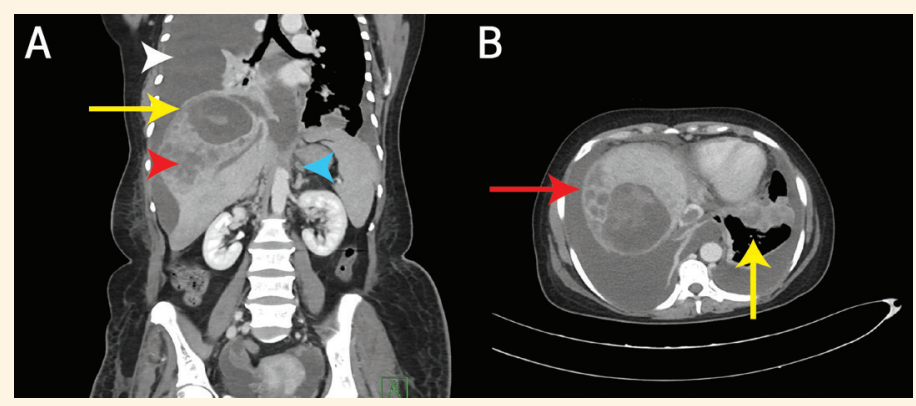

Figure 1: Contrast enhanced computed tomography (CECT) of the abdomen and the thorax. A: Coronal section of CECT abdomen and thorax showing heterogenous hypodense lesion in the right lobe of liver (yellow arrow), possible site of subcapsular rupture (red arrowhead), massive pleural effusion (white arrowhead), inferior vena cava (IVC) compression with hepatic vein thrombosis (blue arrowhead). B: Axial section of CECT abdomen and thorax showing left lower lobe lung heterogenous ill-defined lesion (yellow arrow) and heterogenous hypodense lesion in right lobe of liver with multiple satellite lesions (red arrow).

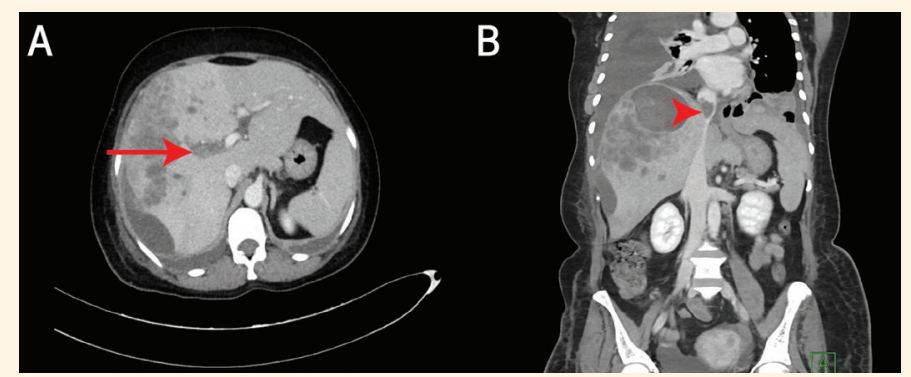

Figure 2: Contrast enhanced computed tomography (CECT) of the abdomen and the thorax. A: Axial section of portal phase CECT of the abdomen and pelvis showing hypodense filling defect in the right branch of the portal vein (arrow). Normally enhancing left branch of the portal vein is seen. B: Coronal reformation of the venous phase of CECT abdomen and pelvis revealing a hypodense filling defect in the supra-hepatic segment of the inferior vena cava suggestive of thrombus (arrowhead).

revealed an ill-defined heterogeneously enhancing lesion in the liver with a communicating subcapsular collection and hypo enhancing lesions in the left lobe and heterogeneously enhancing lesion in the left lung [Figures 1A and B]. The portal vein thrombosis was managed with intravenous heparin as it was an acute thrombosis ( $<60$ days) and thought to be of infective pathology [Figures 2A and B].

Ultrasound-guided aspiration from the subcapsular collection revealed no organism on culture and a fine needle aspiration cytology was carried out from the liver lesion which was nondiagnostic. Pleural fluid aspiration was done twice as a therapeutic procedure and yielded brownish fluid suggestive of sterile necrosis. The aspirate was tested for malignant cytology, but was negative both times [Table 1]. The effusion was attributed to reactive fluid collection secondary to the large liver lesion, as is well documented in large liver lesions of malignant as well as infective pathologies. With CECT revealing a heterogenous ill-defined lesion in the lower lobe of the left lung, a USG-guided core needle biopsy from the lung and liver lesion was carried out which revealed features of adenocarcinoma of the lung with hepatic metastasis [Figure 3]. The patient was managed conservatively with intravenous empirical antibiotics in view of persistent febrile spikes and leukocytosis. She was transferred to the intensive care unit for monitoring and management in view of respiratory distress and desaturation. An intercostal drainage tube was placed in view of the deterioration of tachypnoea, as a palliative procedure for the massive pleural effusion which rapidly reaccumulated despite multiple therapeutic aspirations. The biopsy samples of the lung and liver were sent for assessing epidermal growth factor receptor (EGFR) status and EGFR mutation was found. The patient was started on an oral tyrosine kinase inhibitor, gefitinib. However, the patient's tachypnoea continued to worsen and despite best efforts, there was no response to the treatment and the patient succumbed to the disease. The cause of death was reported as severe respiratory failure and acidosis due to intractable pleural effusion and septicaemia secondary to disseminated lung adenocarcinoma with a ruptured liver metastatic lesion.

Appropriate consent has been obtained from the patient and the attenders for publication of this case. 
Table 1: Description of the radiological and histological characteristics of the current case

\begin{tabular}{|c|c|}
\hline $\begin{array}{l}\text { Radiological } \\
\text { and } \\
\text { histological } \\
\text { Investigations }\end{array}$ & Findings \\
\hline $\begin{array}{l}\text { Chest } \\
\text { radiograph }\end{array}$ & $\begin{array}{l}\text { Bilateral moderate pleural effusion (right } \\
\text { more than left). }\end{array}$ \\
\hline $\begin{array}{l}\text { Ultrasound } \\
\text { abdomen }\end{array}$ & $\begin{array}{l}\text { An ill-defined heterogenous } \\
\text { predominantly hypoechoic collection } \\
\text { in the right lobe of the liver with } \\
\text { no internal vascularity and a large } \\
\text { subcapsular collection around segments } \\
7 \text { and 8, possibly suggestive of a ruptured } \\
\text { pyogenic abscess or hepatocellular } \\
\text { carcinoma. Portal vein showed an } \\
\text { echogenic filling defect in the main } \\
\text { branch extending into the proximal right } \\
\text { and left branches suggestive of portal } \\
\text { venous thrombosis. }\end{array}$ \\
\hline $\begin{array}{l}\text { Contrast } \\
\text { enhanced } \\
\text { computed } \\
\text { tomography } \\
\text { abdomen and } \\
\text { thorax }\end{array}$ & $\begin{array}{l}\text { An } 8.5 \times 7.5 \times 6.5 \mathrm{~cm} \text { ill-defined } \\
\text { heterogeneously enhancing lesion } \\
\text { with fluid attenuating areas and hypo } \\
\text { enhancing areas in segments } 7 \text { and } 8 \\
\text { of the liver with hyperdense contents } \\
\text { within. This lesion was communicating } \\
\text { with a subcapsular collection with } \\
\text { a thickness of } 2.7 \mathrm{~cm} \text {, overlying } \\
\text { the segments } 6 \text { and } 7 \text {, suggesting } \\
\text { a subcapsular rupture. Multiple, } \\
\text { clustered, ill-defined and a few discrete } \\
\text { heterogeneously hypo-enhancing lesions } \\
\text { were noted surrounding the above lesion } \\
\text { and two similar hypo enhancing lesions } \\
\text { were noted in the left lobe of the liver, } \\
\text { around } 2 \times 2 \text { cm. No significant washout } \\
\text { of contrast was noted on venous or } \\
\text { delayed phases. Portal and hepatic } \\
\text { venous thrombosis was noted, but there } \\
\text { was no evidence of direct extension of } \\
\text { the lesion into the veins, suggesting a } \\
\text { benign thrombosis. Right massive pleural } \\
\text { effusion with collapse of right middle } \\
\text { and lower lobes was noted, but there } \\
\text { was no direct extension of tumour into } \\
\text { the pleural cavity; left moderate pleural } \\
\text { effusion was also noted. A clustered } \\
\text { heterogeneously hypo- enhancing lesion } \\
\text { with lobulated margin measuring } 3.6 \\
\times 5 \times 3 \text { cm was noted in the anterior } \\
\text { and lateral basal segments of lower lobe } \\
\text { of the left lung, which was possibly } \\
\text { infective and was suggested for biopsy } \\
\text { correlation. A suspicion of infective or } \\
\text { malignant pathology was considered. }\end{array}$ \\
\hline $\begin{array}{l}\text { Core-needle } \\
\text { biopsy from the } \\
\text { lung lesion }\end{array}$ & $\begin{array}{l}\text { Features of adenocarcinoma of the lung } \\
\text { with focal areas of bronchoalveolar } \\
\text { pattern of spread and occasional foci } \\
\text { of necrosis with tumour cells staining } \\
\text { positive for EGFR. }\end{array}$ \\
\hline $\begin{array}{l}\text { Core-needle } \\
\text { biopsy from the } \\
\text { liver lesion }\end{array}$ & $\begin{array}{l}\text { A malignant, possibly metastatic } \\
\text { tumour focally infiltrating the hepatic } \\
\text { parenchyma displaying abundant } \\
\text { cytoplasm, pleomorphic nuclei and brisk } \\
\text { mitotic activity. Immunohistochemistry } \\
\text { showed positive CK-7 and TTF-1 } \\
\text { suggesting the lesion to be metastases } \\
\text { from a primary in the lung. CK-20, } \\
\text { estrogen receptor and glypican } 3 \text { were } \\
\text { found to be negative. }\end{array}$ \\
\hline $\begin{array}{l}\text { Cytology from } \\
\text { the pleural fluid }\end{array}$ & Negative for malignancy. \\
\hline
\end{tabular}

EGFR = epidermal growth factor receptor; $C K=$ cytokeratin $; T F=$ thyroid transcription factor.

\section{Discussion}

We report a case of carcinoma of the lung with ruptured liver metastasis which led to a diagnostic dilemma due to a dubious presentation mimicking a liver abscess. Although carcinoma of the lung is frequently accompanied by liver metastasis, the incidence of rupture is scarce. ${ }^{7}$ The mechanism of spontaneous rupture of hepatic metastasis is not clear but there are several factors reported in the literature such as tumour necrosis, vascularity, location (subcapsular) and impaired coagulation. ${ }^{7,8}$ In the present case, the probable cause of rupture may be the subcapsular location and necrotic hepatic metastasis which may be due to the presence of portal vein thrombosis.

The commonly observed symptoms in patients with ruptured liver metastasis are nonspecific abdominal pain, discomfort followed by haemodynamic instabilities, fever in case of infected collection or as a part of the systemic inflammatory response (SIRS), acute liver failure and multi-organ dysfunction syndrome. ${ }^{8}$ In the present case, the patient presented with fever, non-specific abdominal pain, SIRS (heart rate $>90$ beats/min and respiratory rate $>20 / \mathrm{min}$ ) and a soft and tender mass in the right hypochondrium.

Abdominal USG is the initial investigation carried out followed by CECT which is ideal in differentiating $\mathrm{HCC}$, liver metastasis and liver abscess. But it has its limitation in hypervascular metastasis. Peripheral location, discontinuity of the hepatic surface with surrounding haemoperitoneum are the characteristic CT findings in a ruptured HCC, which can be extrapolated to liver metastasis as well. ${ }^{4}$ Similarly, in the present case CT revealed multiple liver lesions with subcapsular collection from primary in the lung. The left lower lobe pulmonary lesion visualised on CT was not characteristic of lung carcinoma but was later proven by pathological examination to be the primary. The patient gave a history of firewood usage for cooking purposes. Indoor air pollution with medium particulate matter especially due to usage of biomass fuel has been noted to be an independent risk factor for lung adenocarcinoma in non-smokers. ${ }^{9,10}$

Portal vein thrombosis is infrequently associated with HCC and can be due to tumour extension or due to benign thrombosis in cirrhosis or prothrombotic states. It causes liver hypovascularity and may induce necrosis in the tumour. Piscaglia et al. reported that portal vein thrombosis in a patient with known hepatic malignancy should be appropriately investigated for benign versus malignant cause for thrombosis. ${ }^{11}$ Patients with benign thrombus can be considered 

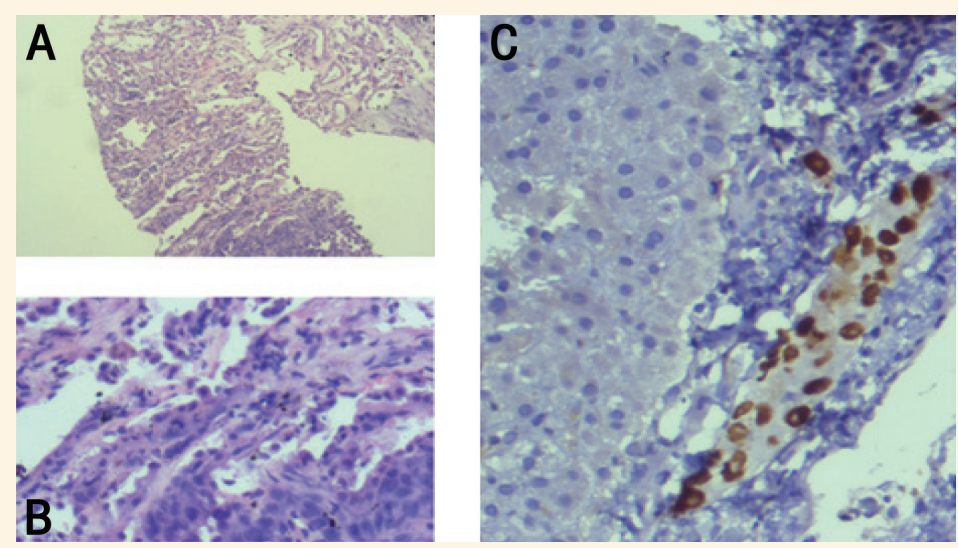

Figure 3: Histopathology of lung and liver lesion. A: Haematoxylin and eosin stain (H\&E) at $\times 40$ magnification showing a core of lung parenchyma with tumor cells in the lower part of the core arising from the alveolar wall forming a bronchioloalveolar pattern and infiltrating the lung parenchyma. B: H\&E stain at $\times 200$ magnification showing lung parenchyma infiltrated by tumor cells arising in glandular pattern exhibiting moderate nuclear atypia and pleomorphism. C: Diaminobenzidine with haematoxylin counterstain at $\times 400$ magnification showing liver parenchyma infiltrated by metastatic deposits of adenocarcinoma from lung, highlighted by thyroid transcription factor-1 (Immunohistochemistry with DAKO monoclonal antibody, USA).

for hepatic transplant whereas malignant thrombosis is a contraindication. Hepatic venous thrombosis leading to Budd-Chiari syndrome is most commonly caused by hypercoagulable states and can lead to acute hepatic necrosis and failure. It is very rarely seen due to neoplasms of the liver and can be a poor prognosis for the patient. In the current case, the metastatic liver lesion had concomitant portal venous and hepatic venous thrombosis which is an extremely rare occurrence and may have contributed to the significant areas of necrosis and possibly the rupture..$^{12,13}$

Diagnosis of non-small cell lung carcinoma is based on histopathology with immunohistochemical markers. Treatment of spontaneous rupture of hepatic metastasis depends on the tumour as well as patient factors such as the tumour size, location, severity of bleeding, active bleeding and haemodynamic stability of the patient., ${ }^{3,14}$ There are various treatment modalities available for ruptured liver metastasis ranging from conservative to surgical resection. A staged approach was advocated in a recent report where the active bleeding is managed by less invasive trans-arterial angioembolisation (TAE), hepatic wedge resection, lobectomy or suture ligation of the bleeder followed by hepatic resection. ${ }^{14}$ Conservative management of ruptured liver metastasis focuses on correcting coagulopathy, close-monitoring and stabilisation of the patient followed by imaging to confirm haemostasis. There are conflicting studies regarding the conservative management in the literature. A retrospective study from Taiwan reported a higher survival rate at 30 days in patients undergoing immediate hepatectomy or a two-staged procedure (TAE followed by hepatectomy) compared to conservative management. ${ }^{15}$ On the contrary, a retrospective study on patients with ruptured HCC and a case report on ruptured liver metastasis in a pancreatic adenocarcinoma were managed successfully with conservative management. ${ }^{14,16}$ Similarly, in the present case, the patient was managed conservatively but had a poor outcome.

Although several treatment options are available in the management of ruptured liver metastasis, the prognosis seems to be poorer in these cases. Ruptured liver metastasis is detrimental in a patient with lung cancer; therefore, early diagnosis with a risk assessment for rupture, close monitoring for high-risk candidates with a selective intervention based on tumour biology and patient factors are the key components for the management of these cases.

\section{Conclusion}

Spontaneous rupture of the liver metastasis is rare, even rarer in a case of adenocarcinoma of the lung. Close monitoring by risk assessment, especially in subcapsular liver secondaries is warranted for early diagnosis and prompt management in case of rupture. More studies are required to find a suitable treatment in such cases. The physician has to decide between a two-staged approach or conservative management based on the patient's haemodynamic stability, tumour characteristics and availability of resources. This report emphasises the importance of the knowledge of rare possible complications of this common condition in order to avoid a diagnostic dilemma. 


\section{AUTHOR CONTRIBUTIONS}

VK conceptualised the work. SM, BG and KSJ conducted the literature review. SM, BG, RNG and KSJ collected and processed the data. BG, SS, AA RNG and KSJ contributed to data analysis and interpretation. SM, BG and KSJ drafted the manuscript. VK, SS, AA and RNG supervised the work and critically reviewed the manuscript. All authors approved the final version of the manuscript.

\section{References}

1. Riihimäki M, Hemminki A, Fallah M, Thomsen H, Sundquist K, Sundquist J, et al. Metastatic sites and survival in lung cancer. Lung Cancer 2014; 86:78-84. https://doi.org/10.1016/j.lung can.2014.07.020.

2. Morgensztern D, Ng SH, Gao F, Govindan R. Trends in Stage Distribution for Patients with Non-small Cell Lung Cancer: A National Cancer Database Survey. J Thorac Oncol 2010; 5:29-33. https://doi.org/10.1097/JTO.0b013e3181c5920c.

3. Gulati A, Vyas S, Lal A, Harsha S, Gupta V, Nijhawan R, et al. Spontaneous rupture of hepatic metastasis from choriocarcinoma: A review of imaging and management. Ann Hepatol 2009; 8:384-7.

4. Choi BG, Park SH, Byun JY, Jung SE, Choi KH, Han J-Y. The findings of ruptured hepatocellular carcinoma on helical CT. Br J Radiol 2001; 74:142-6. https://doi.org/10.1259/ bjr.74.878.740142

5. Chen Z-Y. Etiology and management of hemmorrhage in spontaneous liver rupture: a report of 70 cases. World J Gastroenterol 2002; 8:1063-6. https://doi.org/10.3748/wjg. v8.i6.1063

6. Rajala K, Lehto JT, Sutinen E, Kautiainen H, Myllärniemi M, Saarto T. mMRC dyspnoea scale indicates impaired quality of life and increased pain in patients with idiopathic pulmonary fibrosis. ERJ Open Res 2017; 3:00084-2017. https://doi. org/10.1183/23120541.00084-2017.
7. Mochimaru T, Minematsu N, OhsawaK, TomomatsuK, MiuraH, Betsuyaku $\mathrm{T}$, et al. Hemoperitoneum secondary to rupture of a hepatic metastasis from small cell lung cancer during chemotherapy: A case with a literature review. Intern Med 2017; 56:695-9. https://doi.org/10.2169/internalmedicine.56.6828.

8. Goto Y, Tobino K, Yoshimine K, Sueyasu T, Okahisa M, Sakabe M, et al. An autopsy case of ruptured liver metastases from small cell lung cancer: A case report and literature review. Respir Med Case Rep 2020; 30:101039. https://doi. org/10.1016/j.rmcr.2020.101039.

9. Mu L, Liu L, Niu R, Zhao B, Shi J, Li Y, et al. Indoor air pollution and risk of lung cancer among Chinese female non-smokers. Cancer Causes Control 2013; 24:439-50. https://doi.org/ 10.1007/s10552-012-0130-8.

10. Behera D, Balamugesh T. Indoor air pollution as a risk factor for lung cancer in women. J Assoc Physicians India 2005; 53:190-2.

11. Piscaglia F, Gianstefani A, Ravaioli M, Golfieri R, Cappelli A, Giampalma E, et al. Criteria for diagnosing benign portal vein thrombosis in the assessment of patients with cirrhosis and hepatocellular carcinoma for liver transplantation. Liver transplantation 2010; 16:658-67. https://doi.org/10.1002/lt.22044.

12. Goto Y, Uchino Y, Sasaki S, Shirahama N, Nomura Y, Akiba J, et al. Complete spontaneous necrosis of hepatocellular carcinoma accompanied by portal vein tumor thrombosis: A case report. Int J Surg Case Rep 2018; 44:220-5. https://doi.org/10.1016/j. ijscr.2018.02.045

13. Sabol TP, Molina M, Wu GY. Thrombotic venous diseases of the liver. J Clin Transl Hepatol 2015; 3:189-94. https://doi.org/ 10.14218/JCTH.2015.00014.

14. Rahul A, Robin F, Adarsh H. Spontaneous rupture of hepatic metastasis from pancreatic adenocarcinoma. Case Rep Oncol Med 2016; 2016:6968534. https://doi.org/10.1155/2016/6968534.

15. Hsueh KC, Fan HL, Chen TW, Chan DC, Yu JC, Tsou SS, et al. Management of spontaneously ruptured hepatocellular carcinoma and hemoperitoneum manifested as acute abdomen in the emergency room. World J Surg 2012; 36:2670-6. https:// doi.org/10.1007/s00268-012-1734-6.

16. Leung KL, Lau WY, Lai PB, Yiu RY, Meng WC, Leow CK. Spontaneous rupture of hepatocellular carcinoma: Conservative management and selective intervention. Arch Surg 1999; 134:1103-7. https://doi.org/10.1001/archsurg.134.10.1103. 